\title{
MULTI-LEVEL ENERGY EFFICIENT IMPROVED UNEQUAL CLUSTERING IN WIRELESS SENSOR NETWORKS
}

\author{
K Thyagarajan \\ Research Scholar, Department of Computer Science and Engineering \\ Rayalaseema University, Kurnool \\ Dr T Bhaskara Reddy \\ Professor, Department of Computer Science and Technology \\ Sri Krishnadevaraya University, Anantapuramu
}

\begin{abstract}
In wireless sensor networks (WSNs), the node distribution in the unequal clustering is rapidly used for distributing the load and increase the network lifetime. In tradition unequal clustering mechanism, the nodes which are nearer to the base station suffers with energy depletion. To overcome this issue, this paper proposes the multilevel unequal clustering algorithm with efficient cluster head selection procedure based on the residual energy and maximum transmission capacity of the nodes. The proposed algorithm uses threshold function to resist the waiting time of the node for receiving the cluster head notification message. The simulation results show the efficiency of the proposed algorithm compared with the counterpart algorithms.
\end{abstract}

Keywords: Multilevel clustering, Wireless sensor networks, Clustering, Energy consumption.

Cite this Article: K Thyagarajan and Dr T Bhaskara Reddy, Multi-Level Energy Efficient Improved Unequal Clustering in Wireless Sensor Networks, International Journal of Computer Engineering and Technology, 10(2), 2019, pp. 205-214.

http://iaeme.com/Home/issue/IJCET?Volume=10\&Issue=2

\section{INTRODUCTION}

The major development in the field of sensor devices led to the development of wireless sensor networks. The applications of WSNs are environmental monitoring, disaster management, defence, health care and many more [1]. The WSNs are having large number of sensor nodes which are deployed in the remote regions to cover wider areas. The functionality of the sensor nodes is to collect the sensing data, process and transmit to the base station. The base station is connected to the internet which publishes the data. In many cases, sensor nodes are having limited battery power. It is difficult to replace the sensor nodes once it is deployed in the region 
after depletion of the power. Therefore, minimizing the energy consumption and improving the network life time is the major factor in WSNs. In [2], many issues are addressed in this research area. The cluster based energy efficient mechanism is one of the approaches to address the energy issue. The sensor nodes are divided in to clusters and forms one cluster head for each group. In some conditions, the sensor nodes with high energy are selected as cluster head [3-4]. Each sensor nodes represents only one single cluster senses the local data and forwards to the cluster head. The cluster head aggregates the collected data and process the data to the base station. Figure 1 shows the wireless sensor network model. Clustering is the suitable mechanism to improve the network lifetime by framing the different groups [5]. The clustering can be done in WSNs by using two different methods. One is centralized approach and another one is distributed approach. In the centralized approach, the selection of cluster head is done by base station as predefined by the defined application. In the distributed approach, the sensor node elects the cluster head based on the algorithm.

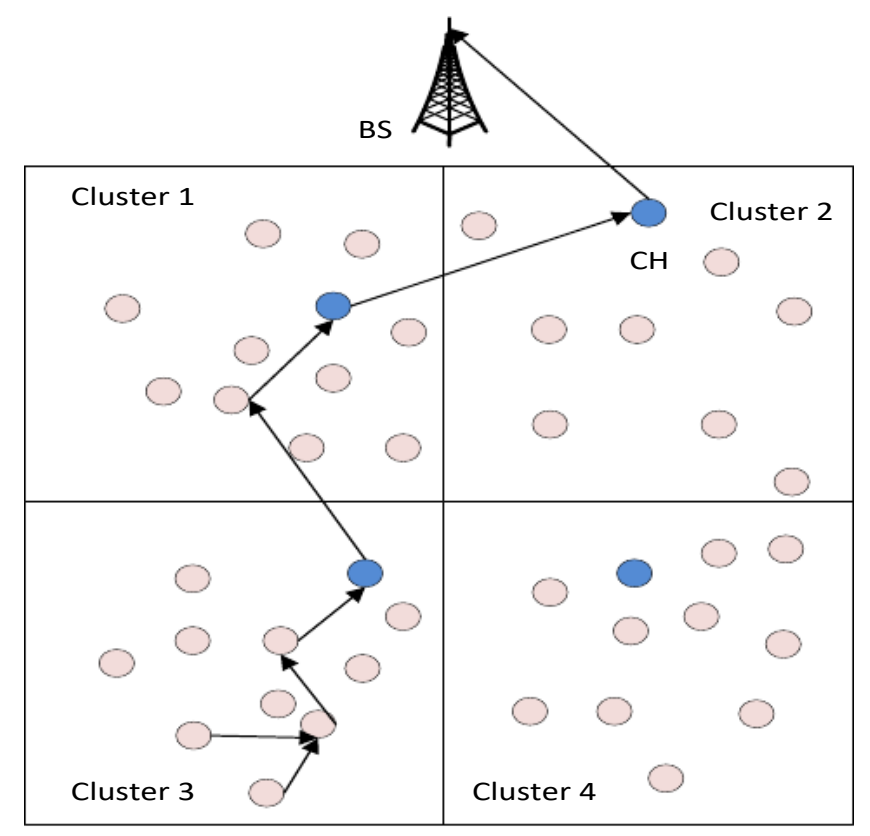

Figure 1 Structure of Wireless Sensor Networks

Many algorithms are proposed with different objectives such as load balancing, fault tolerance, increasing network lifetime and minimizing the data duplications etc. [6]. This paper develops the clustering based energy efficient mechanism for WSNs. The rest of the paper is organized as follows. Section 2 explains about the related work which deeply concentrates on energy efficient routing protocols. Section 3 explains about the network model, energy model and level based mechanism for unequal clustering. Section 4 explains about the proposed energy efficient improved unequal clustering algorithm. Section 5 deals with the simulation analysis and finally the conclusion is drawn in section 6 .

\section{LITERATURE SURVEY}

The main aim of this study is to increase the network lifetime and to decrease the energy consumption of the nodes. The deployment of the nodes in the distributed environment is a challenging task [7]. In [8], the energy efficient algorithms are proposed for cluster based routing. This mechanism uses cluster head rotation process to distribute the consumption of energy over all nodes. The major drawback of this protocol is that there is a chance of selecting the low energy nodes as cluster heads and allows the nodes distribution in the network is not in the uniform manner. In [9], the HEED (Hybrid Energy Efficient Distributed Protocol) was 
proposed to overcome the drawbacks of the LEACH algorithm. The selection of the cluster head is based on the residual energy of the nodes. Therefore, the nodes which having the highest energy will become the cluster heads. The limitation of this protocol is the node which is nearer to the base station will suffer more load due to the incoming packets from the other clusters.

In [10], the authors proposed the unequal clustering algorithm to overcome the limitations of HEED. The main approach of this protocol is to restrict the cluster size which is near to the bases station. This mechanism preserves the energy of the cluster head nearer to the base station. However, the nodes nearer to the BS affected with imposed traffic and increased overhead. In [11], weight based rotation mechanism (WEERC) was proposed to select the cluster heads. This mechanism consists of two steps. As an initial step, the $\mathrm{CH}$ candidates are selected based on the parameters such as degree of the node, transmission range and residual energy. In the next step, the $\mathrm{CH}$ candidates compete with in themselves to become final cluster head based on the available neighbouring nodes with in the transmission range. The proposed routing algorithm uses the rotation process with in the cluster. This process will continue until all the nodes drain their energy.

In [12], the weight driven cluster head selection process was proposed to select the cluster head. The cluster head selection is based on the three metrics such as node distance, frequency of cluster head and remaining energy. The cluster head which falls short below the threshold will be removed as the $\mathrm{CH}$ and the next node which having the highest weight become the cluster head. In [13], the authors proposed dynamic load balancing protocol for WSNs. This protocol selects the $\mathrm{CH}$ based on the highest remaining energy with in the network. The clusters formations in the network are formed in unequal manner. If the $\mathrm{CH}$ energy reached below the threshold, the base station rearranges the cluster of the network. In [14], the hybrid routing protocol is proposed by combining rotation protocol and HEED protocol. This protocol uses rotation mechanism inside the cluster to select the $\mathrm{CH}$.

\section{PRELIMINARIES}

\subsection{Network Model}

This paper considers the following assumptions: $\mathrm{X}$ sensor nodes are deployed randomly in to $\mathrm{K}^{*} \mathrm{~K}$ dimension region. The Base station is situated outside the sensing region. The $\mathrm{x}_{\mathrm{i}}$ nodes are acted as cluster heads where $\mathrm{x}_{\mathrm{i}} \in \mathrm{X}, \mathrm{M}$ clusters are formed and $\mathrm{x}_{\mathrm{i}} \in \mathrm{M}$, the $\mathrm{M}$ are defined as $\mathrm{M}=\left(\mathrm{M}_{1}, \mathrm{M}_{2}, \mathrm{M}_{3} . . \mathrm{M}_{\mathrm{n}}\right)$. The following are the network assumptions for the base station and sensor nodes.

- The sensor nodes deployed in the network are heterogeneous in terms of energy, but they have similar processing capacity, computation capacity and communication capacity.

- The sensor nodes have the energy constraint but not for the base station.

- The sensor nodes are fixed throughout the lifetime of the network and randomly deployed in the regions.

- All sensor nodes are available within the range of the base station to form the clustering process.

\subsection{Energy Consumption Model}

To evaluate the energy consumption of the network, we used the Heinzelman et al. [15] model. Figure 2 shows the energy consumption model of the communication. 


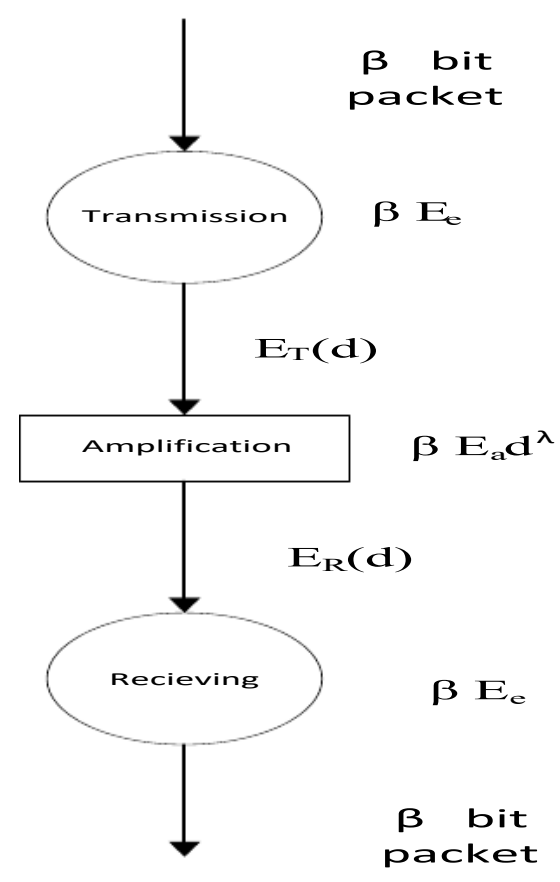

Figure 1 Energy Consumption model of the communication

The transmission energy for $\beta$ bit packet for a distance of $d$ between two nodes is given by equation 1.

$$
\begin{gathered}
E_{\text {Tran }}(\beta, d)=\left\{\begin{array}{l}
\beta E_{e}+\beta \varepsilon_{f} d^{2}, \text { where } d<d_{0} \\
\beta E_{e}+\beta \varepsilon_{a} d^{4}, \text { where } d>d_{0}
\end{array}\right. \\
d_{0}=\sqrt{\frac{\varepsilon_{f}}{\varepsilon_{a}}}
\end{gathered}
$$

The receiving energy for $\beta$ bit packet is given by equation 3 .

$$
E_{\operatorname{Re} c}(\beta)=\beta E_{e}
$$

Where $\beta$ is the number of bits, $E_{\text {Tran }}$ is the transmission energy and $E_{R e c}$ is the receiving energy, $\mathrm{d}$ is the distance between the nodes. $\mathrm{E}_{\mathrm{e}}, \mathrm{E}_{\mathrm{f}}, \mathrm{E}_{\mathrm{a}}$ are the coefficients of the energy consumption model.

\section{PROPOSED ALGORITHM}

\subsection{Unequal Clustering Algorithm for WSNs}

In WSNs, the energy limitation is the major concern to increase the network lifetime. To overcome this issue, energy consumption of the nodes must be managed efficiently. The property of the cluster head is to manage the energy consumption of the nodes in homogeneous networks. Though, there are many algorithms addressed the energy issue in WSNs, still it is not completely achieved. The rotation of cluster heads somewhat balances the energy consumption of the CHs and member nodes and hardly fulfils the objective in the inter-cluster communication. The nodes nearer to the base station deplete the energy quickly due to the packets coming from the nodes located far from the base station. 
The clusters around the base station should be in smaller size. This is due to the CHs consumes high energy with in these clusters. Therefore, more number of clusters needs to be formed around the base station. The formation of the clusters nearer to the base station is done by considering range of the node's competition radius. Consider that $\mathrm{L}_{\max }$ is the highest computation radius of the node which is directly proportional to the distance between the base station and competition node. Let node ' $i$ ' is the competing node for $\mathrm{CH}$ and it calculates the competing radius $L_{i}$ by considering the distance from the base station which is given in $\mathrm{Eq} 4$.

$$
L_{i}=\left(1-\lambda \times \frac{d_{\max }-d\left(x_{i}, B S\right)}{d_{\max }-d_{\min }}\right) \times L_{\max }
$$

Where $d_{\min }$ is the distance from the node which is located nearer to the base station, $d_{\max }$ is the distance from the node which is located farthest from the base station. $\lambda$ is the constant value which lies in between 0 and $1, d\left(x_{i}, B S\right)$ is the distance from the node ' $\mathrm{i}$ ' to the base station.

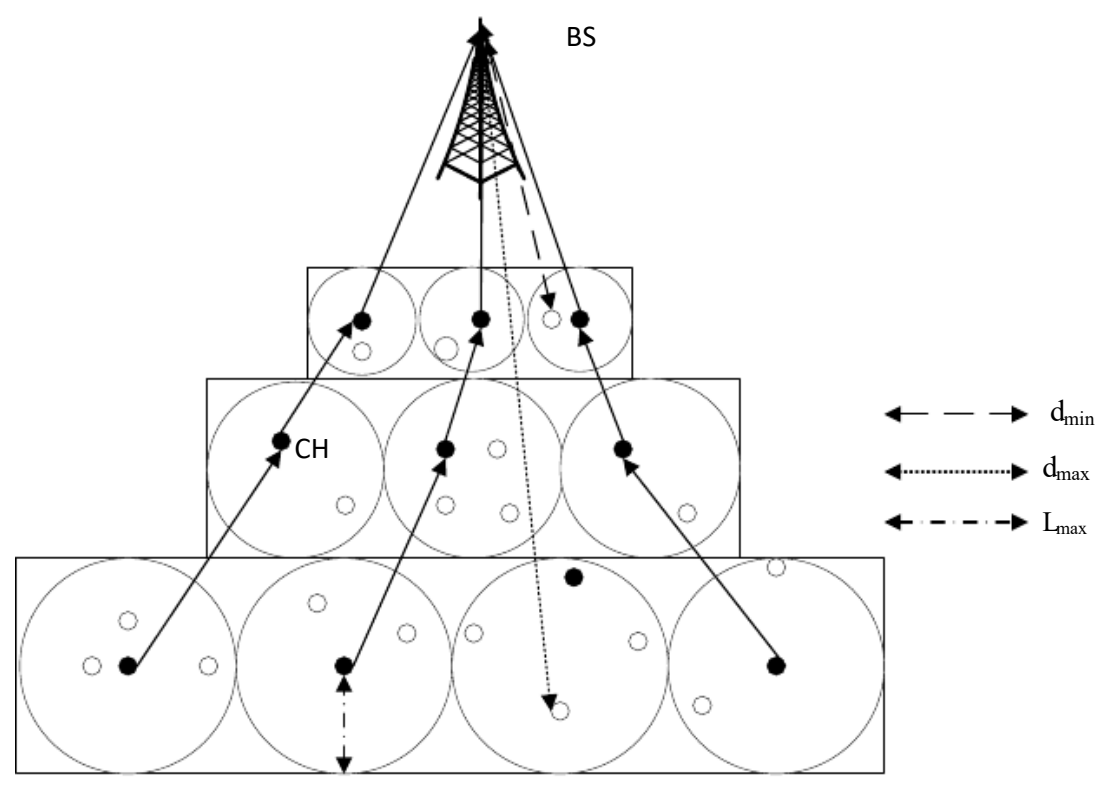

Figure 2 Overview of the proposed algorithm

From the Figure 2, it is observed that the nodes are placed in the different regions of the network. The transmission range of the network is given as $T_{\max }$ and $T_{\min }$. The node needs to find their levels in the network by applying the Eq 5 .

$$
x_{\text {level }}=\frac{d\left(x_{i}, B S\right)-X_{T \max }}{X_{T \max }-T_{\min }}
$$

At the time of cluster head selection, each node forwards their residual energy to the neighbouring nodes with in the radius. The node which is having the highest residual energy is selected as $\mathrm{CH}$ for particular round. This process will avoids the selection of two or more cluster heads in the same cluster. However, the cluster head selection process consists of two stages. In the first stage, all nodes broadcast the bid to become the cluster head. In the second stage, the nodes select the cluster head based highest residual energy and shares the information to the other nodes with in the transmission radius. 


\subsection{Energy Efficient Improved Unequal Clustering Algorithm (EEIUC)}

The proposed Energy Efficient Improved Unequal Clustering Algorithm removes the first step in $\mathrm{CH}$ selection process performed in general procedure of unequal clustering algorithm.

In the initial stage, the base station performs the first step by broadcasting the NEW_MSG which contains the $d_{\min }$ and $d_{\max }$. Every node in the cluster has to forward the message and the time limit for receiving the message is given in Eq. 6.

$$
\tau(i)=\eta \times \tau_{\text {delay }}
$$

Here $\eta$ is the random number which lies in between 0 and $1, \tau_{\text {delay }}$ is the time delay to receive the message from the other nodes. At the initial round, the energy of all nodes is in equal level. Therefore $\eta$ can be set in between 0 and $\tau_{\text {delay. }}$. The node which doesn't receive the NOTIFY_MSG before the $\tau_{\text {delay }}$ expires, it claims himself as the $\mathrm{CH}$ to the cluster for the initial round and forwards the information to all the neighbouring nodes with in the transmission range which has been given in Eq. 4. This mechanism reduces the exchange of messages between the nodes at the time of cluster formation.

The sensor nodes in the network finds the cluster head and sends the JOIN_MSG. For the

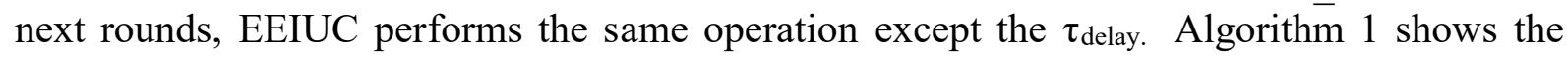
cluster formation in EEIUC algorithm.

Algorithm 1: Cluster formation in EEIUC algorithm

Input: BS_ID $<-$ Base Station ID,

$\mathrm{T}_{\max }<-$ maximum transmission range

$\mathrm{T}_{\min }<-$ minimum transmission range

Begin

1: Base station broadcasts the MSG with information of BS_ID

2: for each $\mathrm{x} \in \mathrm{X}$ do

3: $\quad \mathrm{x}<-\mathrm{d}\left(\mathrm{x}_{\mathrm{i}}, \mathrm{BS}\right)$

4: $\quad \mathrm{x}<-$ Xlevel by computing Eq. 5

4: end for

5 if $\mathrm{x}_{\text {level }}<$ threshold then

6: $\mathrm{x}_{\mathrm{i}}<-$ Level1

7: else

8: $\mathrm{x}_{\mathrm{i}}<-$ Next level

9: end if

End

Algorithm 2 shows the cluster head selection process where each node competes to the position of cluster head by sending the residual energy information to the all nodes with in the transmission range.

Algorithm 2: Cluster Head Selection Algorithm

Begin

1: BS Broadcasts the NEW_MSG

2: $\mathrm{X}_{\mathrm{i}}$ receives the $\mathrm{NEW}$ _MSG and initiates timer $\tau(\mathrm{i})$ based on their residual energy 
3: if $x_{i}$ receives NOTIFY_MSG before $\tau(\mathrm{i})$ expires then

4: $\quad \mathrm{x}_{\mathrm{i}}$ cancels $\tau(\mathrm{i})$ and waits for another nodes NOTIFY_MSG

5: else

6: $\quad \mathrm{x}_{\mathrm{i}}$ declares itself as the $\mathrm{CH}$ and broadcasts the NOTIFY_MSG to all nodes

7: end if

8: if $\mathrm{x}_{\mathrm{i}}$ is not selected as the cluster head then

9: $\quad \mathrm{x}_{\mathrm{i}}$ joins the cluster based on the algorithm 1

10: else

11: $\mathrm{x}_{\mathrm{i}}$ waits until it receives JOIN_MSG

12: end if

End

\section{SIMULATION SETUP}

The simulation is carried out using the Qual Net 6.1 [16] simulator. 200 nodes are deployed in the region of $1000 \times 1000 \mathrm{~m}^{2}$. The nodes are distributed non-uniformly in the network. Table 1 shows the parameters for the simulation. To identify the effectiveness of the proposed protocol, we considered the HEED [9] and EC [17] protocols.

Table 1 Parameters for simulation of EEIUC algorithm

\begin{tabular}{|c|c|}
\hline Parameter & Value \\
\hline No. of Nodes & 200 \\
\hline Network Region $\left(\mathrm{m}^{2}\right)$ & $1000 \times 1000$ \\
\hline Packet Size (bytes) & 200 \\
\hline Initial Energy of nodes $(\mathrm{J})$ & $0.5-1.5$ \\
\hline $\mathrm{E}_{\mathrm{e}}(\mathrm{nJ} / \mathrm{bit})$ & 50 \\
\hline $\mathrm{E}_{\mathrm{a}}\left(\mathrm{pJ} / \mathrm{bit} / \mathrm{m}^{4}\right)$ & 0.0013 \\
\hline $\mathrm{E}_{\mathrm{f}}\left(\mathrm{pJ} / \mathrm{bit} / \mathrm{m}^{2}\right)$ & 10 \\
\hline $\mathrm{L}_{\max }(\mathrm{m})$ & 110 \\
\hline
\end{tabular}

The results of the simulation are carried out with several metrics which are given below:

- Average energy consumption: This evaluation metric is defined as the all nodes average energy consumption in the network for single round.

- Network residual energy: This is as the total residual energy of the network for single round.

- Network Life time: This is defined as the time taken for first node dies in the network.

- Number of nodes alive: This shows how many numbers of nodes are alive in the network after simulation time is completed.

\subsection{Energy Consumption}

The energy consumption of the EEIUC protocol is compared with HEED and EC protocols. Figure 3 shows the average energy consumption of the network for single round. It is observed that the energy consumption of the EEIUC protocol is slightly less compared to the HEED and EC protocols. It is due to the proposed EEIUC eliminates the first step in the process of $\mathrm{CH}$ selection by avoiding the broadcasting of bids to elect as $\mathrm{CH}$. The HEED and EC follows the bid procedure for the selection of Ch. Therefore, the average energy consumption of the network of EEIUC is less. 


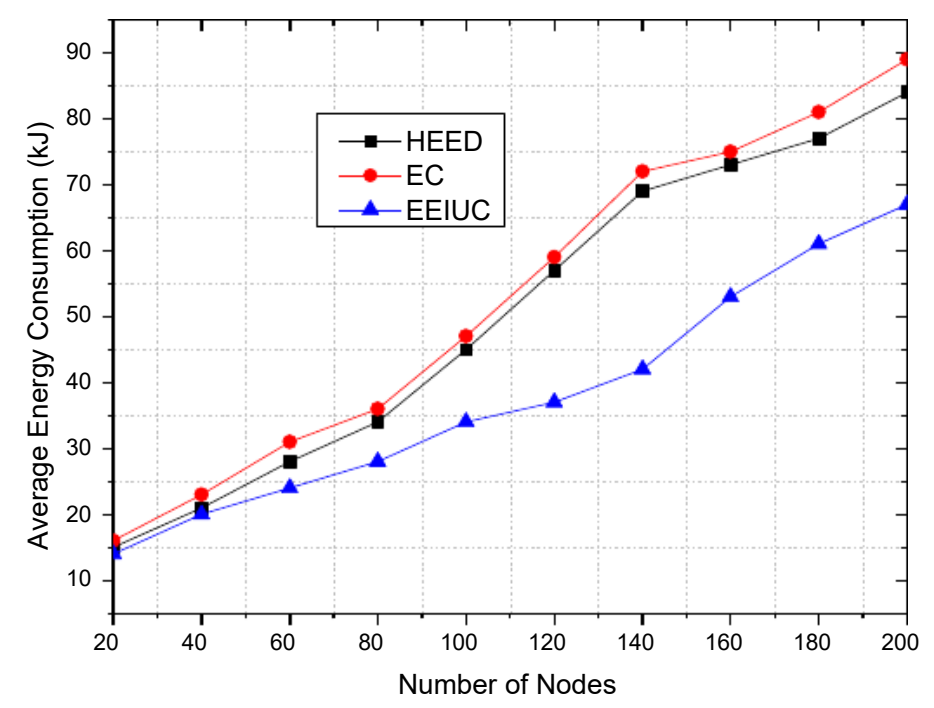

Figure 3 Average energy Consumption Vs Number of Nodes

\subsection{Network Residual Energy}

Figure 4 represent the average residual energy of the network in EEIUC with respect to the number of simulation rounds. It is identifying that the residual energy of the nodes in the network is reduces at the common rate in EEIUC, HEED and EC protocols. In EEIUC, the nodes which are present in the layer 1 consume more energy due to their presence nearer to the BS. The other protocols such as HEED and EC also consume more energy due to their network behaviour.

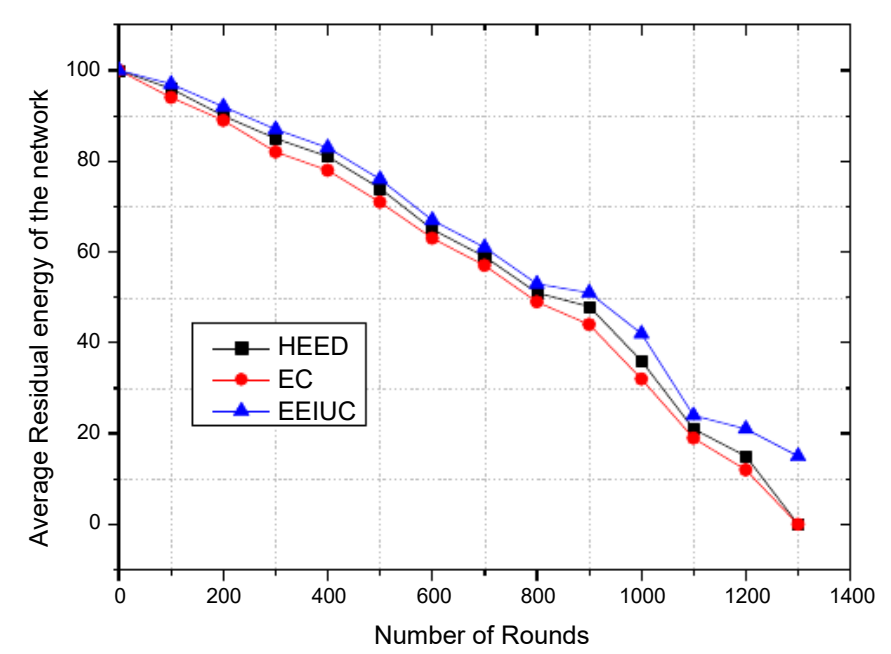

Figure 4 Average Residual Energy of the Network Vs Number of Rounds

\subsection{Network Life time}

Figure 5 shows the network life time in EEIUC, HEED and EC protocols. It is observed that the Network lifetime of EEIUC is higher compared to the HEED and EC protocol. The proposed EEIUC protocol uses time delay mechanism to select the $\mathrm{CH}$. It reduces the waiting time and 
energy consumption of the node compared to the HEED and EC protocol. Therefore, the residual energy of the nodes will be high.

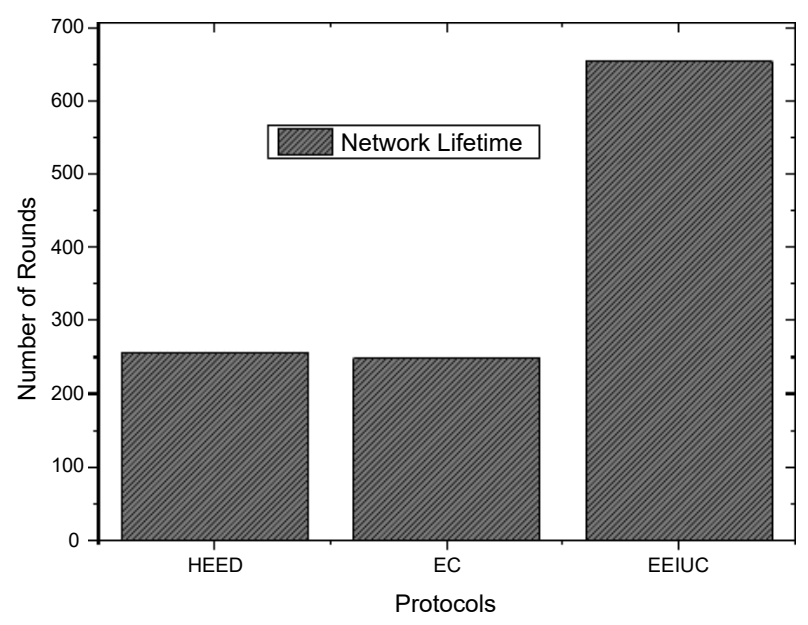

Figure 5 Network life time in EEIUC, HEED and EC when first node dies

\subsection{Number of Nodes Alive}

Figure 6 shows the number of nodes alive when the number of rounds progress in EEIUC, HEED and EC. It is observed that the EEIUC shown improved performance compared to the remaining protocols. EEIUC recorded 18\% improvement in terms of number of nodes alive compared to the HEED and 21\% improvement compared to the EC routing protocol.

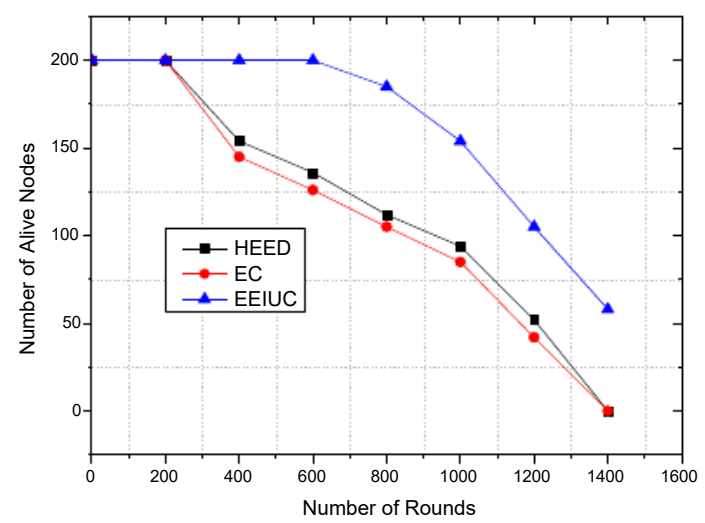

Figure 6 Number of Alive Nodes Vs Number of Rounds

\section{CONCLUSION}

This paper presents the Energy Efficient Improved Unequal Clustering Algorithm (EEIUC) for wireless sensor networks. The proposed algorithms eliminate the procedure of bidding for the selection of $\mathrm{CH}$ in the cluster. EEIUC allows the level based mechanism to improve the lifetime of the network by managing the nodes which are nearer to the base station. The simulation of the proposed protocol is carried out using the four metrics such as energy consumption, network lifetime, number of alive nodes and network residual energy. The proposed protocol proved their efficiency while comparing with HEED and EC protocols. 


\section{REFERENCES}

[1] Rao, PC Srinivasa, and Haider Banka. "Novel chemical reaction optimization based unequal clustering and routing algorithms for wireless sensor networks." Wireless Networks23, no. 3 (2017): 759-778.

[2] Sabor, Nabil, Mohammed Abo-Zahhad, Shigenobu Sasaki, and Sabah M. Ahmed. "An unequal multi-hop balanced immune clustering protocol for wireless sensor networks." Applied Soft Computing 43 (2016): 372-389.

[3] Xia, Hui, Rui-hua Zhang, Jia Yu, and Zhen-kuan Pan. "Energy-efficient routing algorithm based on unequal clustering and connected graph in wireless sensor networks." International Journal of Wireless Information Networks 23, no. 2 (2016): 141150 .

[4] Wu, Dapeng, Jing He, Honggang Wang, Chonggang Wang, and Ruyan Wang. "A hierarchical packet forwarding mechanism for energy harvesting wireless sensor networks." IEEE Communications Magazine 53, no. 8 (2015): 92-98.

[5] Zhang, De-gan, Si Liu, Ting Zhang, and Zhao Liang. "Novel unequal clustering routing protocol considering energy balancing based on network partition \& distance for mobile education." Journal of Network and Computer Applications 88 (2017): 1-9.

[6] Malathi, L., R. K. Gnanamurthy, and Krishnan Chandrasekaran. "Energy efficient data collection through hybrid unequal clustering for wireless sensor networks." Computers \& Electrical Engineering 48 (2015): 358-370.

[7] Gupta, Vrinda, and Rajoo Pandey. "An improved energy aware distributed unequal clustering protocol for heterogeneous wireless sensor networks." Engineering Science and Technology, an International Journal 19, no. 2 (2016): 1050-1058.

[8] Wu, Dapeng, Jing He, Honggang Wang, Chonggang Wang, and Ruyan Wang. "A hierarchical packet forwarding mechanism for energy harvesting wireless sensor networks." IEEE Communications Magazine 53, no. 8 (2015): 92-98.

[9] O. Younis and S. Fahmy. "HEED: A Hybrid, Energy-Efficient,Distributed Clustering Approach for Ad Hoc Sensor Networks," IEEE Trans. Mobile Computing, vol. 3, no. 4, Oct 2014, pp. 366-79.

[10] Darabkh, Khalid A., Noor J. Al-Maaitah, Iyad F. Jafar, and Khalifeh Ala'F. "Energy efficient clustering algorithm for wireless sensor networks." In 2017 International Conference on Wireless Communications, Signal Processing and Networking (WiSPNET), pp. 590-594. IEEE, 2017.

[11] Huang, Jianhua, Yadong Hong, Ziming Zhao, and Yubo Yuan. "An energy-efficient multihop routing protocol based on grid clustering for wireless sensor networks." Cluster Computing20, no. 4 (2017): 3071-3083.

[12] Wu, Dapeng, Jing He, Honggang Wang, Chonggang Wang, and Ruyan Wang. "A hierarchical packet forwarding mechanism for energy harvesting wireless sensor networks." IEEE Communications Magazine 53, no. 8 (2015): 92-98.

[13] Sharma, Neha, and Kanika Sharma. "Energy Efficient Clustering Based on Expectation Maximization for Homogeneous Wireless Sensor Network." Energy 6, no. 8 (2017).

[14] Aierken, Nueraili, Roberto Gagliardi, Leonardo Mostarda, and Zaib Ullah. "RUHEEDrotated unequal clustering algorithm for wireless sensor networks." In 2015 IEEE 29th International Conference on Advanced Information Networking and Applications Workshops, pp. 170-174. IEEE, 2015.

[15] W.B. Heinzelman, A. Chandrakasan, H. Balakrishnan, An application-specific protocol architecture for wireless microsensor networks, IEEE Trans. Wirel. Commun. 1 (4) (2002) 660-670.

[16] https://www.scalable-networks.com/qualnet-network-simulation accessed in 21/12/2018.

[17] D. Wei et al., "An Energy-Efficient Clustering Solution for Wireless Sensor Networks," IEEE Trans. Wireless Commun., vol. 10, no. 11, Sept. 2011, pp. 3973-83. 